Published in final edited form as:

Trends Immunol. 2017 November ; 38(11): 858-871. doi:10.1016/j.it.2017.06.002.

\title{
Regulation of the Immune System by Laminins
}

\author{
Thomas Simon and Jonathan S. Bromberg \\ Departments of Surgery and Microbiology and Immunology, Center for Vascular and Inflammatory \\ Disease, University of Maryland School of Medicine
}

\begin{abstract}
Laminins are trimeric proteins that are major components of the basement membranes that separate endothelia and epithelia from the underlying tissue. Sixteen laminin isoforms have been described, each with distinct tissue expression patterns and functions. While laminins have a critical structural role, recent evidence also indicates that they also impact the migration and functions of immune cells. Laminins are differentially expressed upon immunity or tolerance and orientate the immune response. This review will summarize the structure of laminins, the modulation of their expression, and their interactions with the immune system. Finally, the role of the laminins in autoimmune diseases and transplantation will be discussed.
\end{abstract}

\section{Basement Membranes and Laminins}

\begin{abstract}
Basement membranes (BMs) are cell-associated extracellular matrix (ECM) protein networks that cover the basal side of epithelial and endothelial cells. BMs are essential for tissue formation and cell differentiation. Their composition varies depending of the tissues, but laminins, collagens, nidogen, proteoglycans, and agrin are the main components. Laminins are large molecular weight trimeric glycoproteins composed of three different disulfide-linked polypeptides, termed $a, \beta$, and $\gamma$ (Figure 1). Laminins have an essential role in the formation of BMs and in the maintenance of their integrity. Indeed, due to their interaction with specific cell surface receptors such as integrins, laminins allow endothelial cell anchorage to the BM. The interaction of laminins with other components of the BM such as nidogen, perlecan, or laminins themselves promotes BM assembly and integrity of the BM (Figure 2) [1,2]. Several isoforms exist for each of the three polypeptides that compose laminins, and the association between these three chains can generate 16 distinct laminin isoforms (Table 1). Every BM contains at least one laminin isoform, and the laminin isoforms present in the BM dictate its architecture and properties. Genetic ablation in animal models or mutations in humans that eliminate laminin expression prevent BM formation and are often lethal or associated with severe physiological disorders (for review see [3]). For instance, the laminin a 4 null mice develop hemorrhage during the embryonic and perinatal
\end{abstract}

\footnotetext{
My contact information: Jonathan S. Bromberg, MD, PhD, Professor of Surgery and Microbiology and Immunology Vice Chair for Research University of Maryland School of Medicine, 22 S. Greene St., S8B06, Baltimore, MD 21201, Office 410-328-6430, FAX 410-328-0401,jbromberg@som.umaryland.edu.

Publisher's Disclaimer: This is a PDF file of an unedited manuscript that has been accepted for publication. As a service to our customers we are providing this early version of the manuscript. The manuscript will undergo copyediting, typesetting, and review of the resulting proof before it is published in its final citable form. Please note that during the production process errors may be discovered which could affect the content, and all legal disclaimers that apply to the journal pertain.
} 
stages, suggesting a central role for this laminin subunit in microvessel integrity [4]. Mouse embryos lacking laminin a5 die late in embryogenesis and exhibit multiple developmental defects with exencephaly and syndactyly, highlighting an essential role in developmental processes [5]. In the lymphoid organs and more specifically in the lymph node, the vascular and lymphatic systems that support immune cell trafficking are surrounded abluminally by BM that contains only two laminin isoforms, laminins 411 and 511 . Thus, to penetrate the vessel walls from the luminal side to the abluminal side, leukocytes need to first interact with endothelial cells, cross the endothelial barrier, and then finally cross the BM where they interact with laminins. Laminins 411 and 511 are also present as part of the ECM in the lymph node cortical ridge, a region that is essential to bring $\mathrm{T}$ lymphocytes and antigenpresenting cells together [6]. It is also important to note that the laminins regulate endothelial cell phenotype and function [7], so that indirectly they also influence leukocyteendothelial interactions. Interestingly, most leukocytes express laminin-specific receptors, suggesting that laminins could regulate leukocyte migration. Moreover, through their receptor binding, laminins regulate multiple signaling pathways that could affect leukocyte proliferation, activation, or survival. Altogether, this suggests that the crosstalk between laminins and immune cells can modulate the immune response in an organ- and contextspecific manner. In this review, we describe the current knowledge on laminin regulation, as well as the impact of laminin binding on immune cells. Finally, we discuss the potential roles of laminins in autoimmune or allogenic responses.

\section{Laminins: Structural Proteins}

\section{Structure and Role}

Laminins are composed of one $a$, one $\beta$, and one $\gamma$ chain and were described and characterized for the first time in 1979 [8,9]. Currently, five $a$, three $\beta$, and three $\gamma$ chains have been defined for a total number of 16 laminin isoforms (Table 1). The different isoforms share a common cross-shaped structure characterized by the separation of the $\mathrm{N}$ terminal portions of the three subunits that form three short arms [10,11] (Figure 1). The short arms are composed of a globular laminin N-terminal domain (LN) and a globular laminin IV domain (L4/LF) [12,13]. Each of these domains is separated by rodlike regions formed by multiple laminin epidermal-growth-factor-like (LE) domains [14]. The a 3A, a4, and $\gamma 2$ subunits have truncated short arms and lack the LN domain. The $a, \beta$, and $\gamma$ subunits are joined together in a triple a-helical coiled-coil domain that forms the long arm $[15,16]$. The short arms allow the interaction with other ECM proteins and laminin selfassembly (Figure 2). Indeed, laminin self-assembly [17] and perlecan [18] binding involve the LN domain of the short arms. Nidogen binds the LE domain of the laminin $\gamma 1$ [19] and $\gamma 3$ [20] chains and the L4 domain of the $\gamma 2$ chain [21]. Nidogen and perlecan allow the laminin network to be connected to collagen IV [22,23]. The C-terminal end is composed of five homologous globular domains (LG domains) specific to a chains [24]. The LG domains are mostly ligands for cell surface integrin and non-integrin-type receptors. Among the integrins that can be expressed by leukocytes (Table 2), the a $3 \beta 1$ and a $6 \beta 4$ integrins have been described to recognize the laminin 332 and 511/521 isoforms. The a $6 \beta 1$ integrin shows broad specificity and interacts with laminins 211/221, 332, 411, and 511, with the highest affinity for laminin 511 [25]. The non- integrin laminin receptor a-dystroglycan can

Trends Immunol. Author manuscript; available in PMC 2018 November 01. 
also bind the LG domain of the laminin $2 a$ [26] and a 5 chains [27]. Although the expression of a-dystroglycan has been described for certain leukocyte populations such as thymocytes [28], the effect of its interaction with laminin on immune cell function is still largely unknown.

The variations among the three-subunit associations endow the various trimers with unique properties and functions. The distribution of the different isoforms is tissue specific (Table 1) and confers unique physiological properties to the BMs. For instance, epithelial BMs express high levels of the laminin 332 isoform that allow the binding of epithelial cells via the a $6 \beta 4$ integrin and the formation of hemidesmosomes. Thus, mutation of any of the three laminin 332 chain genes in humans is associated with junctional epidermolysis bullosa, characterized by a poor dermal-epidermal adhesion [29]. Because of their capacity to bind numerous ECM proteins and surface membrane receptors, laminins form supramolecular networks that are essential to BM integrity and to the correct development of tissues and organs. For these reasons, laminin functions have usually been viewed through the lens of this architectural role and their implications for cell differentiation processes. However, recent data suggest that distinct laminins may specifically modulate many different aspects of immune regulation.

\section{Laminin Expression}

The tissue distribution of the different laminin isoforms is well characterized (Table 1) and the specific cellular origin for each laminin chain has been extensively studied [30-32]. Numerous cell types can produce and secrete laminins. Depending on the tissue, epithelial, endothelial, or mesenchymal cells have the ability to produce laminin subunits. The polymerization of the three chains into a functional trimer is a complex process that occurs at the cell surface and involves interaction with specific cell surface receptors such as integrin and dystroglycan [33]. Regarding the laminin production specifically in the lymphoid organs, various cell types can produce and secrete laminins. Laminins a 2, a 4, and a 5 chains, but not a 1, are found in mouse and human bone marrow [34] and synthesized by stromal cells $[35,36]$. In the thymus, laminin isoforms containing the a 2 chain or the a5 chain are expressed in the subcapsular epithelium and in thymic blood vessels. Expression of the laminin a 4 chain seems to be restricted to endothelial cells of the thymus, whereas the laminin 332 isoform could be detected on medullary thymic epithelial cells and weakly in the subcapsular epithelium [37]. In the spleen [38] and the lymph nodes [6,39], laminins 411 and 511 are the major expressed laminins and are supposed to be produced mainly by fibroblastic reticular cells in the lymph nodes [40]. Fibroblastic reticular cells also express laminin a 2, a 3, and $\gamma 3$ chains. However, the cellular origin for laminins in the secondary lymphoid organs is even more complex as leukocytes can also produce or express these structural proteins. Indeed, macrophages express cell surface laminin [41]. Laminin 411 is secreted by blood neutrophils [42], monocytes [43], platelets [44], and lymphocytes [45]. Lymphocytes can also secrete laminin 511 after stimulation [46]. However, the secretion of laminins 411 and 511 by immune cells is a matter of debate as laminin chain expression seems to be restricted to cytoplasm in these studies and other teams have shown opposite results [47]. Thus, laminin secretion by leukocyte will require further investigations. 
The expression of the laminins is differentially controlled by numerous signals such as growth factors, cytokines, and proteases. Laminin a 4 expression is increased in endothelial cells after treatment with lipopolysaccharide, interleukin-1 $\beta$ (IL-1 $\beta$ ), and tumor necrosis factor- $a$ (TNF- $\alpha$ ) and decreased by the angiostatic agent hydroxymethylprogesterone $[31,48]$. Laminin $\alpha 5$ is also upregulated by TNF- $\alpha$ or IL- $1 \beta$, but hydroxymethylprogesterone treatment increases its expression. Changes in laminin expression have been reported in various inflammatory diseases. Patients with allergic asthma show an increased expression of laminin 511 in the subepithelial BM in the lung compared with healthy individuals [49]. Overexpression of laminin a 5 and a 1 chains has been observed in inflammatory bowel disease [50]. Inflammation associated with ischemic injury increases laminin expression on endothelial cells in the central nervous system [51]. Finally, our laboratory previously demonstrated that laminin expression in the lymph node is differentially regulated following the induction of tolerance versus immunity [6]. In the lymph nodes of tolerant mice treated with allogenic splenocytes and anti-CD40L antibody, laminin $a 4$ is increased, whereas laminin a 5 is overexpressed in immune animals immunized with allogenic splenocytes only. In addition to this modulation of expression, laminins can also be regulated by degradation by proteinases. For instance, the ADAM9 proteinase has the ability to specifically degrade laminin and is overexpressed on activated leukocytes [52]. Altogether, these different mechanisms contribute to the fact that the laminin composition of BMs is both variable among organs and dynamic, for example, during inflammation.

\section{Laminins and Immune System Interactions}

In addition to their architectural role, laminins support and influence the migration and the function of immune cells (Table 2 and Figure 3). Leukocyte recruitment to sites of injury or inflammation relies on sequential interactions of immune cells first with endothelial junction components in the vessel wall and then with the perivascular BM. Among the components of the BM, laminins can act as ligands that bind cell membrane receptors (mainly integrins), initiating integrin-mediated signaling (also referred to as outside-in signaling). However, the precise details of the effects of laminins on immune cells and the molecular pathways involved are largely unknown. Because of the difficulty of analyzing the impact of laminins in vivo, most of the experiments that describe the interaction of laminins with leukocytes have been done in vitro with laminins coated on plates. In contrast to laminin recombinant proteins, the use of laminin isolated from cell lines or placenta raises the question of the purity of such preparations and the potential contamination by other factors such as nidogen or collagen that can impact the function of immune cells.

\section{Immune Cell Progenitors}

Stromal laminins in the bone marrow regulate proliferation, differentiation, and function of hematopoietic stem and progenitor cells. Laminins 211, 411/421, and [34,36]. In vitro, $\mathrm{CD} 4^{+}$hematopoietic 511/521 are present in the bone marrow progenitors adhere strongly via the integrin $a 6 \beta 1$ to laminin 511/521 isolated from placenta and this interaction favors proliferation [35]. The adhesion of $\mathrm{CD} 34^{+}$hematopoietic progenitors to laminin 511/521 and also to recombinant laminin 411 has been confirmed by $\mathrm{Gu}$ and colleagues [53]. They 
also demonstrated that laminins 411 and 511/521 facilitate stromal-derived factor- 1 alphastimulated transmigration of hematopoietic progenitors via integrin a 6 in transwell assay. Blocking integrin a 6 inhibits homing of progenitor cells in vivo into bone marrow and leads to retention of progenitors in the blood [54].

Intrathymic T-cell differentiation relies on progenitor cells released from the bone marrow into the circulation to enter the thymus at the corticomedullary junction via blood vessels. Progenitors then migrate as double negative thymocytes $\left(\mathrm{CD}^{-}{ }^{-} \mathrm{CD} 8^{-}\right)$from the subcapsular area into the cortex, where they undergo maturation into $\mathrm{CD} 4^{+} \mathrm{CD} 8^{+}$thymocytes. The double-positive thymocytes differentiate into single positive cells as they migrate from the cortex toward the medulla. Each location of the thymus expresses specific laminin isoforms that could dictate thymocyte migration and maturation. Laminins 211 and 511 are present in the BM of the thymic blood vessels [55]. Compared with mature thymocytes, doublenegative thymocytes bind preferentially via integrin a $6 \beta 1$ to these laminin isoforms isolated from placenta [37,56], and thus these laminins may favor the entry of immature thymocytes at the corticomedullary junction. These laminins are also expressed in the subcapsular epithelium [37] where thymocyte maturation starts. Laminin 211 has pleiotropic effects on thymocytes as laminin a 2 deficiency (dy/dy mice) is associated with architectural disorganization of the thymus correlated with a dramatic reduction in the size of the doublepositive thymocyte population [57]. In vitro, laminin 211 isolated from placenta favors the proliferation of anti-CD3-activated thymocytes via the integrins $\alpha 3 \beta 1$ and $\alpha 6 \beta 1$ [58]. This laminin isoform seems to regulate apoptosis as an increased number of apoptotic thymocytes has been observed in the thymic capsular and subcapsular regions in dy/dy mice [57] and increased apopototic cell death of the double-positive thymocytes in laminin a2-chain null mutant mice dy3k/dy3k [59]. The absence of laminin 211 has no effect on the apoptosis of mature single-positive thymocytes. The double-positive thymocytes in the cortex lose the ability to adhere to laminin 511 and then migrate into the medulla where laminin 332 is highly expressed [37]. In transwell Boyden chambers, laminin 332 isolated from the SSC25 cell line promotes the migration of mature thymocytes via integrins $\alpha 3 \beta 1$ and $\alpha 6 \beta 4$ [60]. In this assay, the interaction of mature thymocytes with laminin 332 induces the activation of matrix metalloproteinase-14 (MMP-14) that releases a soluble fragment of CD44. This soluble fragment increases thymocyte migration in the transwell chamber [60]. Taken together, these data suggest that laminins in the thymus play important functions in the development of thymocytes into functional mature $\mathrm{T}$ lymphocytes.

\section{Innate Immune Cells}

Laminins also interact with many innate immune cells, such as neutrophils [61,62]. In vitro experiments with coated laminin 111 isolated from Engelbreth-Holm-Swarm (EHS) sarcoma cells revealed that the adhesion of neutrophils to laminin is mediated by integrin a $6 \beta 1[63,64]$, and enhances TNF- $\alpha$ production [65] and macrophage inflammatory protein-1 $\beta$ (MIP-1 $\beta$ ) secretion [66]. Laminin 111 isolated from EHS sarcoma cells stimulates neutrophil lysozyme release and superoxide production in response to chemoattractants [67] and enhances bactericidal activity [68]. However, while the laminin 111 is ubiquitously expressed in the embryo, its expression in the adult is restricted to a 
small subset of BMs. Thus, this isoform is unlikely to be encountered by any immune cells in vivo during the extravasation process.

In vivo, the recruitment of neutrophils into the peritoneal cavity after an inflammatory stimulus is impaired in laminin a4-deficient mice [42]. Laminin 511 has an important role for the neutrophil migration, and specific regions within the vascular walls show low expression of laminin 511 [7,47]. These sites are preferentially used by transmigrating neutrophils to penetrate the vascular BM. These exit points for emigrating neutrophils are enlarged by neutrophil elastase. Blockade of a 6 integrin or elastase inhibits neutrophil transmigration through the perivascular BM [69]. Neutrophil elastase also cleaves laminin 332 and generates fragments that are chemotactic for neutrophils. However, the role of neutrophil elastase on leukocyte transmigration has not yet been proven in vivo since mice lacking neutrophil elastase have normal extravasation [70].

Laminins promote adhesion of basophils [71,72] and eosinophils [73,74] via integrin a $6 \beta 1$ in vitro. Adhesion of eosinophils favors their viability. Laminins reduce the activation and degranulation of eosinophils stimulated with platelet-activating factor, C5a, or IL-5 [75]. However, all these studies were performed with laminins isolated from placenta, so the role of specific laminin isoforms remains to be determined.

Laminins 332 isolated from the SSC25 cell line and laminin 511 isolated from placenta favor mast cell adhesion and migration via integrin a $3 \beta 1$ [76]. Laminin 332, but not 511, enhances secretion of IL- 8 in the presence of an IgE stimulus. In contrast to mature mast cells, immature mast cells express a $6 \beta 1$ integrin and adhere to laminin 111 isolated from EHS sarcoma cells [77,78]. TGF- $\beta$ treatment induces integrin a 7 [79] and allows mature mast cells to adhere to laminin 111 .

\section{Antigen-Presenting Cells}

Macrophage adhesion to laminin 111 isolated from EHS sarcoma cells seems limited to activated macrophages [80,81] and is mediated by integrin a $6 \beta 1$ [82]. Laminins 111, 411, and 511 have no effect on the transmigration of activated primary macrophages in response to CCL3 in vitro [83]. However, addition of laminin 111 isolated from EHS sarcoma cells to macrophage or monocyte cultures enhances their phagocytic function [84], and upregulates macrophage urokinase-type plasminogen activator and MMP-9 expression [85], mediated by a specific peptide sequence present in laminin a 1 and a 5 subunits [86]. This cryptic peptide is also chemotactic for neutrophils and macrophages, and increases TNF-a production and TNF receptor expression by macrophages [87]. However, it is not clear whether the laminin a 5 sequence that has been shown in vitro to have chemotactic effects is indeed exposed in the intact molecule. Given the stability of this molecule to proteases and the absence of proteolytic activity associated with sites of extravasation, it is debated whether such a peptide is relevant for the in vivo extravasation process. Incubation of monocytes with laminin 111 also slightly increases the production of TNF- $a$, IL-1 $\beta$, IL-6, and MMP-9 [88]. Laminin 111 enhances granulocyte macrophage colony-stimulating factor signaling in monocytic cell lines by increasing granulocyte macrophage colony-stimulating factorinduced mitogen-activated protein kinase (MAPK) phosphorylation and Stat5 phosphorylation [65]. Monocyte adhesion to laminin 111 (from EHS cells) and to 
recombinant laminins 411 and 511 (from placenta) is mediated by integrins a $6 \beta 1$ and $\beta 2$ [43], and laminin 411 but not 511 promotes migration of monocytes.

Laminin 511 from placenta increases the phagocytic function of monocyte-derived dendritic cells (DCs) but also decreases maturation marker expression [89]. Immature bone marrowderived DCs bind laminin 511 via integrin $\beta 1$ and the adhesion reduces their motility in vitro [39]. Laminin 411 (purified from preadipocyte cell lines) or 211 (purified from mouse heart) is not adhesive for immature DCs and laminin 111 (EHS cells) is even repulsive. By contrast, mature DCs do not bind to these four laminins. Bone marrow-derived DCs and CD34+-derived DCs bind to laminin 332 [90]. The close relation of immature DCs with laminins was confirmed in the lymph node, as resident DCs during steady-state conditions show a direct interaction with laminins, whereas mature DCs immigrating during inflammatory conditions show significantly less contact [39].

In vivo, we demonstrated that blocking the integrin-binding site of laminin a 5 favors the migration of plasmacytoid DCs (pDCs) in the LN [6]. In accordance with this result, the inhibition of laminin a 5 degradation with an MMP inhibitor increases the amount of laminin 511 in the LN and reduces the migration of pDCs. Inhibition of laminin a 4 integrin binding also reduces $\mathrm{pDC}$ migration in the $\mathrm{LN}$. Together, these results demonstrate that laminin a5 impedes while a 4 permits pDC migration in the LN through the endothelium and associated BM.

\section{Lymphocytes}

In the spleen marginal zone (MZ), B-lymphocyte location coincides precisely with laminin a5-positive areas [38]. B-cell depletion decreases laminin a5 MZ expression, suggesting that B-cell presence may contribute to the maintenance of laminin a 5 expression. B lymphocytes bind to laminin a 5 via a 6 and $\beta 1$ integrins and the genetic depletion of laminin a5 in the $\mathrm{MZ}$ reduces B-cell content. Transient retention of newly formed B cells in the $\mathrm{MZ}$ via integrin a $6 \beta 1$-laminin a5-mediated interactions promotes $\mathrm{MZ}$ B-cell formation and their survival.

Geberhiwot and colleagues [45] demonstrated that $\mathrm{CD}^{+}{ }^{+} \mathrm{T}$ cells bind via a $6 \beta 1$ integrin to recombinant laminins 411 and 511 isolated from placenta. Both laminins favor proliferation of CD4 ${ }^{+}$T cells activated with immobilized CD3 antibody [45]. They also demonstrated that laminin 411 but not 511 promotes $\mathrm{CD}^{+}{ }^{+} \mathrm{T}$-cell migration in transwell assays although the effect is small and variable. In a second study [46], the same team showed that only laminin 511 (recombinant protein or from placenta) supports T-cell adhesion and migration and promotes T-cell proliferation. Others showed that unstimulated T cells ( $\mathrm{T}$ cell line) adhered weakly to laminin 511 (from placenta) and exhibited minimal binding to laminin 411 (from $3 \mathrm{~T} 3$ and preadipocyte cell lines) [48]. By contrast, phorbol myristate acetate-stimulated T cells bind efficiently to laminin 511 via integrin a $6 \beta 1$ and only weakly to laminin 411 . Discrepancies among the reports may be explained by the use of poorly characterized laminin preparations isolated from human placenta versus recombinant laminin proteins, variation in the laminin concentrations, and also use of unpurified lymphocyte populations or T-cell lines. Thus, future investigations must define precisely the roles of laminins 411 and 511 in $\mathrm{CD}^{+}$and $\mathrm{CD}^{+}$T-cell functions. We recently demonstrated that highly purified 
recombinant laminin 511 inhibits $\mathrm{CD} 4^{+} \mathrm{T}$-cell transendothelial migration toward CCL21 in vitro [6]. Real-time microscopy showed that T-cell movements over endothelial cells are hindered by laminin 511 . By contrast, laminin a 4 increases T-cell transendothelial migration. These data demonstrate that laminin a 4 is more permissive for movement on and into the endothelial cell layer, while laminin a 5 prevents such movements. This study confirms previous in vivo observations that T-cell transmigration through endothelial BMs occurs when laminin a 4 is present and when laminin a 5 is weakly expressed $[48,83]$. In laminin a4-deficient mice, the infiltration of T lymphocytes into inflamed tissue is reduced [83]. This study which was the first one to show that migration of in vivo-activated $\mathrm{T}$ cells is inhibited specifically by laminin 511 also showed for the first time that chemokine-driven transmigration of $\mathrm{T}$ lymphocytes across laminin 511-coated filters is reduced compared with laminin 411-coated filters. Finally, as demonstrated for pDCs, blocking the integrin-binding site of laminin a 5 favors the migration of regulatory T cells in the LN [6], while inhibition of laminin a4 integrin-binding function reduces it.

Together, these studies suggest that laminin 511 restricts leukocyte transendothelial migration while laminin 411 is more permissive. However, in vitro migration assays without endothelial cell monolayers show that leukocytes migrate similarly across laminins 411 and 511. This suggests that in vivo the laminins do more than just directly affecting leukocyte migration and likely alter endothelial cell properties. This has been confirmed recently by Song and colleagues [7] who showed that laminin 511 via integrins $\beta 1$ and $\beta 3$ increased endothelial junctional tightness by stabilizing vascular endothelial-cadherin and downregulating CD99L2, and inhibited leukocyte transmigration. Thus, the modulation of laminin expression or the blocking of laminin interaction with receptors may be an attractive way to regulate leukocyte migration in vivo.

\section{Role of Laminins in Autoimmune Diseases and Transplantation}

\section{Autoimmunity}

The migration of autoimmune cells in inflamed tissue is regulated by laminins. In a model of experimental autoimmune encephalomyelitis (EAE), Wu and colleagues [83] showed that encephalitogenic T-lymphocyte interaction with endothelial BM laminins represents a key rate-limiting step in the initial stages of EAE development. T-lymphocyte extravasation correlates with sites expressing laminin a 4 and small amounts of laminin a5. Genetic elimination of laminin a 4 or integrin a $6 \beta 1$ resulted in reduced pathogenic T-cell and antigen-presenting cell infiltration into the central nervous system and thereby reduced EAE severity. In mice lacking laminin a 4, laminin a 5 is ubiquitously expressed along the vascular tree. These results suggest that direct interaction between infiltrating $\mathrm{CD} 4^{+} \mathrm{T}$ lymphocytes and laminin 411 in the endothelial BM via the integrin a $6 \beta 1$ facilitates extravasation and that laminin 511 inhibits it. Melanoma cell adhesion molecule (MCAM) is specifically expressed by Th17 T cells and is also a receptor for laminin 411 [91]. AntiMCAM monoclonal antibody inhibits T-cell adhesion to laminin 411, reduces Th17 cell infiltration into the central nervous system, and decreases EAE incidence. Thus, targeting Tlymphocyte interactions with endothelial laminins might be a new therapeutic strategy to inhibit pathogenic immune cell infiltration into inflamed organs in autoimmune diseases. 
Laminins are also targets of autoimmunity. Laminin 332 has been identified as an autoantigen in various skin autoimmune diseases. Patients with cicatricial pemphigoid, an autoimmune subepithelial blistering disease, have IgG antilaminin 332 autoantibodies [92]. Injection in mice of antilaminin 332 antibodies mimics this pathology by inducing subepithelial blisters. Antibodies against laminin $\gamma 1$ have been detected in patients suffering from antilaminin $\gamma 1$ pemphigoid, formerly known as anti-p200 pemphigoid [93]. The pathogenicity of antilaminin $\gamma 1$ autoantibodies has been confirmed ex vivo. Indeed, these antibodies interfere with the binding of nidogen to laminin and so disrupt the BM structure and can activate granulocytes and the complement cascade [94]. Autoantibodies against laminin 332, laminin 111, or laminin $\gamma 1$ have also been reported in patients with lupus erythematosus [95-97]. Similar autoantibody-mediated mechanisms occur in rheumatic heart disease and autoimmune myocarditis [98]. Laminins can also drive T-cell-mediated autoimmune responses. Peripheral human $\mathrm{CD}^{+}$and $\mathrm{CD}^{+} \mathrm{T}$-cell clones isolated from a rheumatic carditis patient show reactivity against laminin [99]. Laminin might also be key autoantigens in psoriasis, inducing a T-cell-mediated autoimmune response [100]. The relationship of laminins to autoimmunity may be a consequence of their ubiquitous presence and interaction with many components of the immune system. The pathological consequences of antilaminin antibodies also suggest that development of pharmacologic modulators of laminins will require careful consideration and evaluation to avoid toxicities.

\section{Transplantation}

As described earlier, one of the main roles for laminins is to regulate the migration of immune cells. This has important repercussions for transplantation. In rodent models of allogenic cardiac transplantation, graft rejection is associated with a higher expression of laminins in the graft that precedes cellular infiltration in the graft tissue [101]. Increases in soluble laminin levels in the plasma have been used as indicators of acute rejection in rat small bowel allografts [102], and for human liver transplantation [103]. Local treatment with antilaminin antibody decreases leukocyte cardiac allograft infiltration and the inflammatory cytokines interferon- $\gamma$, TNF- $a$, and IL-2 [104]. These results suggest that inflammation is associated with laminin overexpression in the graft that favors leukocyte infiltration and graft rejection.

The role of the laminins in transplant survival is not only limited to their expression in the graft but also in the lymph nodes that serve as integral sites for the control of immunity and tolerance. We recently demonstrated that induction of immunity by immunization with allogenic splenocytes favors laminin 511 expression in the lymph node cortical ridge [6]. By contrast, induction of tolerance by immunization with allogenic splenocytes and anti-CD40L antibody is associated with relatively higher expression of laminin 411 compared with laminin 511. Treatment of cardiac-transplanted animals with antilaminin a 5 antibody favors Treg and tolerogenic pDC migration into the lymph node and prolongs graft survival. Inhibition of laminin 511 degradation or treatment with antilaminin a 4 antibody has opposite effects. Indeed, blocking laminin 411 circumvents tolerance induction and induces cardiac allograft inflammation and rejection. Inhibition of T-cell binding to laminin 511 is sufficient to alter T-cell migratory patterns and prolongs graft function in rejecting allograft recipients. These findings suggest remodeling of laminin 511 in the lymph node as a

Trends Immunol. Author manuscript; available in PMC 2018 November 01. 
molecular control driving the generation of immunity versus tolerance, and laminin 411 remodeling as a necessary step in tolerance induction. Together, these studies showed that laminin proteins represent a target for acutely generating transplant tolerance or enhancing immunogenicity of antigens.

\section{Concluding Remarks: Laminins As Therapeutic Targets}

As described previously, laminins and especially the isoforms 411 and 511 have pleiotropic effects on the immune system (Figure 3). These laminins are not only passive structural molecules, but also act as molecular switches for tolerance and immunity. Whereas the specific role of these two laminins in the modulation of leukocyte functions will need more investigations in the future with highly purified laminin isoforms and cell populations in vitro and use of laminin a chain-conditional knockout mice in vivo, it seems clear that laminins 411 and 511 have opposites effects. For these reasons, laminins appear as interesting targets for the modulation of the immune response in vivo. Antilaminin antibodies showed encouraging results in the modulation of the immune response [6] but can also be associated with severe biological disorders [105]. Thus, more investigations will be necessary to carefully evaluate the long-term effect of laminin-modifying treatments. Another approach is to target the laminin receptors and the best candidate seems to be, for now, the integrin a $6 \beta 1$ (Table 2). However, numerous other receptors can mediate the laminin effects, including members of the integrin family or non-integrin receptors such as the $67-\mathrm{kDa}$ receptor or a-dystroglycan. The diversity of the laminin receptors may also explain the different effects on the immune system of the various laminin isoforms and represent potential candidates for therapeutic interventions in the future (see Outstanding Questions).

\section{References}

1. McKee KK, et al. Role of laminin terminal globular domains in basement membrane assembly. J Biol Chem. 2007; 282:21437-21447. [PubMed: 17517882]

2. Li S, et al. Laminin-sulfatide binding initiates basement membrane assembly and enables receptor signaling in Schwann cells and fibroblasts. J Cell Biol. 2005; 169:179-189. [PubMed: 15824137]

3. Yao Y. Laminin: loss-of-function studies. Cell Mol Life Sci. 2017; 74:1095-1115. [PubMed: 27696112]

4. Thyboll J, et al. Deletion of the laminin alpha4 chain leads to impaired microvessel maturation. Mol Cell Biol. 2002; 22:1194-1202. [PubMed: 11809810]

5. Miner JH, et al. Roles for laminin in embryogenesis: exencephaly, syndactyly, and placentopathy in mice lacking the laminin alpha5 chain. J Cell Biol. 1998; 143:1713-1723. [PubMed: 9852162]

6. Warren KJ, et al. Laminins affect T cell trafficking and allograft fate. J Clin Invest. 2014; 124:22042218. [PubMed: 24691446]

7. Song J, et al. Endothelial basement membrane laminin 511 contributes to endothelial junctional tightness and thereby inhibits leukocyte transmigration. Cell Rep. 2017; 18:1256-1269. [PubMed: 28147279]

8. Timpl R, et al. Laminin - a glycoprotein from basement membranes. J Biol Chem. 1979; 254:99339937. [PubMed: 114518]

9. Rohde $\mathrm{H}$, et al. Immunochemical characterization of the basement membrane glycoprotein laminin. Eur J Biochem. 1979; 102:195-201. [PubMed: 391564] 
10. Engel J, et al. Shapes, domain organizations and flexibility of laminin and fibronectin, two multifunctional proteins of the extracellular matrix. J Mol Biol. 1981; 150:97-120. [PubMed: 6795355]

11. Bruch M, et al. Dissection of laminin by cathepsin G into its long-arm and short-arm structures and localization of regions involved in calcium dependent stabilization and self-association. Eur $\mathbf{J}$ Biochem. 1989; 185:271-279. [PubMed: 2511014]

12. Hussain SA, et al. Determinants of laminin polymerization revealed by the structure of the a 5 chain amino-terminal region. EMBO Rep. 2011; 12:276-282. [PubMed: 21311558]

13. Carafoli F, et al. Crystal structures of the network-forming short-arm tips of the laminin $\beta 1$ and $\gamma 1$ chains. PLoS One. 2012; 7:e42473. [PubMed: 22860131]

14. Stetefeld J, et al. Crystal structure of three consecutive laminin-type epidermal growth factor-like (LE) modules of laminin gamma1 chain harboring the nidogen binding site. J Mol Biol. 1996; 257:644-657. [PubMed: 8648630]

15. Barlow DP, et al. Sequencing of laminin B chain cDNAs reveals C-terminal regions of coiled-coil alpha-helix. EMBO J. 1984; 3:2355-2362. [PubMed: 6209134]

16. Paulsson M, et al. Evidence for coiled-coil alpha-helical regions in the long arm of laminin. EMBO J. 1985; 4:309-316. [PubMed: 3848400]

17. Schittny, Yurchenco PD. Terminal short arm domains of basement membrane laminin are critical for its self-assembly. J Cell Biol. 1990; 110:825-832. [PubMed: 2307709]

18. Garbe JH, et al. Complete sequence, recombinant analysis and binding to laminins and sulphated ligands of the N-terminal domains of laminin alpha3B and alpha5 chains. Biochem J. 2002; 362:213-221. [PubMed: 11829758]

19. Pöschl E, et al. Site-directed mutagenesis and structural interpretation of the nidogen binding site of the laminin gammal chain. EMBO J. 1996; 15:5154-5159. [PubMed: 8895559]

20. Gersdorff N, et al. Laminin gamma3 chain binds to nidogen and is located in murine basement membranes. J Biol Chem. 2005; 280:22146-22153. [PubMed: 15824114]

21. Sasaki T, et al. Short arm region of laminin-5 gamma2 chain: structure, mechanism of processing and binding to heparin and proteins. J Mol Biol. 2001; 314:751-763. [PubMed: 11733994]

22. Aumailley M, et al. Binding of nidogen and the laminin-nidogen complex to basement membrane collagen type IV. Eur J Biochem. 1989; 184:241-248. [PubMed: 2506015]

23. Kleinman HK, et al. Formation of a supramolecular complex is involved in the reconstitution of basement membrane components. Biochemistry. 1983; 22:4969-4974. [PubMed: 6227336]

24. Timpl R, et al. Structure and function of laminin LG modules. Matrix Biol. 2000; 19:309-317. [PubMed: 10963991]

25. Nishiuchi R, et al. Ligand-binding specificities of laminin-binding integrins: a comprehensive survey of laminin-integrin interactions using recombinant alpha3beta1, alpha6beta1, alpha7beta1 and alpha6beta4 integrins. Matrix Biol. 2006; 25:189-197. [PubMed: 16413178]

26. Tisi D, et al. Structure of the C-terminal laminin G-like domain pair of the laminin a 2 chain harbouring binding sites for a-dystroglycan and heparin. EMBO J. 2000; 19:1432-1440. [PubMed: 10747011]

27. Yu H, Talts JF. Beta1 integrin and alpha-dystroglycan binding sites are localized to different laminin-G-domain-like (LG) modules within the laminin alpha5 chain G domain. Biochem J. 2003; 371:289-299. [PubMed: 12519075]

28. Liou LY, et al. Functional glycosylation of dystroglycan is crucial for thymocyte development in the mouse. PLoS One. 2010; 5:e9915. [PubMed: 20369005]

29. Pulkkinen L, et al. Mutations in the $\gamma 2$ chain gene (LAMC2) of kalinin/laminin 5 in the junctional forms of epidermolysis bullosa. Nat Genet. 1994; 6:293-298. [PubMed: 8012393]

30. Sorokin L, et al. Expression of novel 400-kDa laminin chains by mouse and bovine endothelial cells. Eur J Biochem. 1994; 223:603-610. [PubMed: 8055931]

31. Frieser M, et al. Cloning of the mouse laminin alpha 4 cDNA. Expression in a subset of endothelium. Eur J Biochem. 1997; 246:727-735. [PubMed: 9219532]

32. Sorokin LM, et al. Developmental regulation of the laminin alpha5 chain suggests a role in epithelial and endothelial cell maturation. Dev Biol. 1997; 189:285-300. [PubMed: 9299121] 
33. Colognato H, et al. Laminin polymerization induces a receptor- cytoskeleton network. J Cell Biol. 1999; 145:619-631. [PubMed: 10225961]

34. Gu Y, et al. Characterization of bone marrow laminins and identification of alpha5-containing laminins as adhesive proteins for multipotent hematopoietic FDCP-Mix cells. Blood. 1999; 93:2533-2542. [PubMed: 10194432]

35. Siler U, et al. Characterization and functional analysis of laminin isoforms in human bone marrow. Blood. 2000; 96:4194-4203. [PubMed: 11110691]

36. Siler U, et al. Laminin gamma 2 chain as a stromal cell marker of the human bone marrow microenvironment. Br J Haematol. 2002; 119:212-220. [PubMed: 12358928]

37. Kutlesa $S$, et al. Developmentally regulated interactions of human thymocytes with different laminin isoforms. Immunology. 2002; 105:407-418. [PubMed: 11985661]

38. Song J, et al. Extracellular matrix of secondary lymphoid organs impacts on B-cell fate and survival. Proc Natl Acad Sci U S A. 2013; 110:E2915-E2924. [PubMed: 23847204]

39. Sixt M, et al. The conduit system transports soluble antigens from the afferent lymph to resident dendritic cells in the T cell area of the lymph node. Immunity. 2005; 22:19-29. [PubMed: 15664156]

40. Malhotra D, et al. Transcriptional profiling of stroma from inflamed and resting lymph nodes defines immunological hallmarks. Nat Immunol. 2012; 13:499-510. [PubMed: 22466668]

41. Wicha MS, Huard TK. Macrophages express cell surface laminin. Exp Cell Res. 1983; 143:475479. [PubMed: 6339258]

42. Wondimu Z, et al. An endothelial laminin isoform, laminin 8 (alpha4beta1gamma1), is secreted by blood neutrophils, promotes neutrophil migration and extravasation, and protects neutrophils from apoptosis. Blood. 2004; 104:1859-1866. [PubMed: 15172971]

43. Pedraza C, et al. Monocytic cells synthesize, adhere to, and migrate on laminin- 8 (alpha 4 beta 1 gamma 1). J Immunol 1950. 2000; 165:5831-5838.

44. Nigatu A, et al. Megakaryocytic cells synthesize and platelets secrete alpha5-laminins, and the endothelial laminin isoform laminin 10 (alpha5beta1gamma1) strongly promotes adhesion but not activation of platelets. Thromb Haemost. 2006; 95:85-93. [PubMed: 16543966]

45. Geberhiwot T, et al. Laminin-8 (alpha4beta1gamma1) is synthesized by lymphoid cells, promotes lymphocyte migration and costimulates T cell proliferation. J Cell Sci. 2001; 114:423-433. [PubMed: 11148143]

46. Gorfu G, et al. Laminin isoforms of lymph nodes and predominant role of alpha5-laminin(s) in adhesion and migration of blood lymphocytes. J Leukoc Biol. 2008; 84:701-712. [PubMed: 18523231]

47. Wang S, et al. Venular basement membranes contain specific matrix protein low expression regions that act as exit points for emigrating neutrophils. J Exp Med. 2006; 203:1519-1532. [PubMed: 16754715]

48. Sixt M, et al. Endothelial cell laminin isoforms, laminins 8 and 10, play decisive roles in $\mathrm{T}$ cell recruitment across the blood-brain barrier in experimental autoimmune encephalomyelitis. J Cell Biol. 2001; 153:933-946. [PubMed: 11381080]

49. Amin K, et al. Uncoordinated production of laminin-5 chains in airways epithelium of allergic asthmatics. Respir Res. 2005; 6:110. [PubMed: 16179086]

50. Spenlé $\mathrm{C}$, et al. The Laminin response in inflammatory bowel disease: protection or malignancy? PLoS One. 2014; 9:e111336. [PubMed: 25347196]

51. Ji K, Tsirka SE. Inflammation modulates expression of laminin in the central nervous system following ischemic injury. J Neuroinflammation. 2012; 9:159. [PubMed: 22759265]

52. Roychaudhuri R, et al. ADAM9 is a novel product of polymorphonuclear neutrophils: regulation of expression and contributions to extracellular matrix protein degradation during acute lung injury. $\mathrm{J}$ Immunol. 2014; 193:2469-2482. [PubMed: 25063875]

53. Gu YC, et al. Laminin isoform-specific promotion of adhesion and migration of human bone marrow progenitor cells. Blood. 2003; 101:877-885. [PubMed: 12393739]

54. Qian $\mathrm{H}$, et al. Contribution of alpha6 integrins to hematopoietic stem and progenitor cell homing to bone marrow and collaboration with alpha4 integrins. Blood. 2006; 107:3503-3510. [PubMed: 16439681] 
55. Yousif LF, et al. Laminin isoforms in endothelial and perivascular basement membranes. Cell Adh Migr. 2013; 7:101-110. [PubMed: 23263631]

56. Chang AC, et al. Expression of merosin in the thymus and its interaction with thymocytes. J Immunol. 1993; 151:1789-1801. [PubMed: 8345183]

57. Magner WJ, et al. Aberrant development of thymocytes in mice lacking laminin-2. Dev Immunol. 2000; 7:179-193. [PubMed: 11097211]

58. Chang AC, et al. Alpha 3 beta 1 and alpha 6 beta 1 integrins mediate laminin/merosin binding and function as costimulatory molecules for human thymocyte proliferation. J Immunol. 1995; 154:500-510. [PubMed: 7814863]

59. Iwao M, et al. Interaction of merosin (laminin 2) with very late activation antigen-6 is necessary for the survival of $\mathrm{CD}^{+} \mathrm{CD}^{+}$immature thymocytes. Immunology. 2000; 99:481-488. [PubMed: 10792494]

60. Vivinus-Nebot M, et al. Mature human thymocytes migrate on laminin-5 with activation of metalloproteinase-14 and cleavage of CD44. J Immunol. 2004; 172:1397-1406. [PubMed: 14734715]

61. Bryant G, et al. A role for the laminin receptor in leukocyte chemotaxis. J Leukoc Biol. 1987; 41:220-227. [PubMed: 2951468]

62. Yoon PS, et al. Human neutrophil laminin receptors: activation-dependent receptor expression. J Immunol. 1987; 138:259-265. [PubMed: 2946778]

63. Bohnsack JF, et al. Human neutrophil adherence to laminin in vitro. Evidence for a distinct neutrophil integrin receptor for laminin. J Exp Med. 1990; 171:1221-1237. [PubMed: 1969920]

64. Bohnsack JF. CD11/CD18-independent neutrophil adherence to laminin is mediated by the integrin VLA-6. Blood. 1992; 79:1545-1552. [PubMed: 1347710]

65 . Chen J, et al. The laminin receptor modulates granulocyte-macrophage colony-stimulating factor receptor complex formation and modulates its signaling. Proc Natl Acad Sci U S A. 2003; 100:14000-14005. [PubMed: 14614142]

66. Chiba K, et al. Neutrophils secrete MIP-1 beta after adhesion to laminin contained in basement membrane of blood vessels. Br J Haematol. 2004; 127:592-597. [PubMed: 15566363]

67. Pike MC, et al. Laminin promotes the oxidative burst in human neutrophils via increased chemoattractant receptor expression. J Immunol. 1989; 142:2004-2011. [PubMed: 2537868]

68. Hermann M, et al. Neutrophil bactericidal activity against Staphylococcus aureus adherent on biological surfaces. Surface-bound extracellular matrix proteins activate intracellular killing by oxygen-dependent and -independent mechanisms. J Clin Invest. 1990; 86:942-951. [PubMed: 2394841]

69. Wang S, et al. PECAM-1, alpha6 integrins and neutrophil elastase cooperate in mediating neutrophil transmigration. J Cell Sci. 2005; 118:2067-2076. [PubMed: 15840647]

70. Young RE, et al. Role of neutrophil elastase in LTB4-induced neutrophil transmigration in vivo assessed with a specific inhibitor and neutrophil elastase deficient mice. Br J Pharmacol. 2007; 151:628-637. [PubMed: 17471175]

71. Quan S, et al. Spontaneous and cytokine induced basophil adhesion evaluated by microtiter assay. J Immunol Methods. 2002; 262:121-127. [PubMed: 11983225]

72. Smaniotto $\mathrm{S}$, et al. Mouse basophils reside in extracellular matrix-enriched bone marrow niches which control their motility. PloS One. 2013; 8:e70292. [PubMed: 24086246]

73. Tourkin A, et al. Eosinophil adhesion and maturation is modulated by laminin. Cell Adhes Commun. 1993; 1:161-176. [PubMed: 8081878]

74. Georas SN, et al. Expression of a functional laminin receptor (alpha 6 beta 1, very late activation antigen-6) on human eosinophils. Blood. 1993; 82:2872-2879. [PubMed: 8219235]

75. Kita H, et al. Extracellular matrix proteins attenuate activation and degranulation of stimulated eosinophils. J Immunol. 1996; 156:1174-1181. [PubMed: 8557995]

76. Sime W, et al. Human mast cells adhere to and migrate on epithelial and vascular basement membrane laminins LM-332 and LM-511 via alpha3beta1 integrin. J Immunol. 2009; 183:46574665. [PubMed: 19752234] 
77. Fehlner-Gardiner C, et al. Integrin VLA-6 (alpha 6 beta 1) mediates adhesion of mouse bone marrow-derived mast cells to laminin. Allergy. 1996; 51:650-656. [PubMed: 8899118]

78. Vliagoftis H, Metcalfe DD. Characterization of adhesive interactions between mast cells and laminin isoforms: evidence of a principal role for alpha 6 integrin. Immunology. 1997; 92:553560. [PubMed: 9497498]

79. Rosbottom A, et al. TGF- $\beta 1$ regulates adhesion of mucosal mast cell homologues to laminin-1 through expression of integrin a 7. J Immunol. 2002; 169:5689-5695. [PubMed: 12421948]

80. Mercurio AM, Shaw LM. Macrophage interactions with laminin: PMA selectively induces the adherence and spreading of mouse macrophages on a laminin substratum. J Cell Biol. 1988; 107:1873-1880. [PubMed: 2972733]

81. Shaw LM, Mercurio AM. Interferon gamma and lipopolysaccharide promote macrophage adherence to basement membrane glycoproteins. J Exp Med. 1989; 169:303-308. [PubMed: 2491881]

82. Shaw LM, et al. The activation dependent adhesion of macrophages to laminin involves cytoskeletal anchoring and phosphorylation of the alpha 6 beta 1 integrin. J Cell Biol. 1990; 110:2167-2174. [PubMed: 2141029]

83. Wu C, et al. Endothelial basement membrane laminin a 5 selectively inhibits Tymphocyte extravasation into the brain. Nat Med. 2009; 15:519-527. [PubMed: 19396173]

84. Bohnsack JF, et al. Connective tissue proteins and phagocytic cell function. Laminin enhances complement and Fc-mediated phagocytosis by cultured human macrophages. J Exp Med. 1985; 161:912-923. [PubMed: 3157764]

85. Khan KMF, Falcone DJ. Role of laminin in matrix induction of macrophage urokinase-type plasminogen activator and 92-kDa metalloproteinase expression. J Biol Chem. 1997; 272:82708275. [PubMed: 9079647]

86. Adair-Kirk TL, et al. A site on Laminin a5, AQARSAASKVKVSMKF, induces inflammatory cell production of matrix metalloproteinase-9 and chemotaxis. J Immunol. 2003; 171:398-406. [PubMed: 12817023]

87. Adair-Kirk TL, et al. A chemotactic peptide from laminin alpha 5 functions as a regulator of inflammatory immune responses via TNF alpha-mediated signaling. J Immunol. 2005; 174:16211629. [PubMed: 15661925]

88. Chana RS, et al. Monocyte adhesion to mesangial matrix modulates cytokine and metalloproteinase production. Kidney Int. 2003; 63:889-898. [PubMed: 12631069]

89. García-Nieto S, et al. Laminin and fibronectin treatment leads to generation of dendritic cells with superior endocytic capacity. PLoS One. 2010; 5:e10123. [PubMed: 20419094]

90. Kohl K, et al. Subpopulations of human dendritic cells display a distinct phenotype and bind differentially to proteins of the extracellular matrix. Eur J Cell Biol. 2007; 86:719-730. [PubMed: 17689829]

91. Flanagan K, et al. Laminin-411 is a vascular ligand for MCAM and facilitates TH17 cell entry into the CNS. PloS One. 2012; 7:e40443. [PubMed: 22792325]

92. Lazarova Z, et al. Antiepiligrin cicatricial pemphigoid represents an autoimmune response to subunits present in laminin 5 (alpha3beta3gamma2). Br J Dermatol. 1998; 139:791-797. [PubMed: 9892943]

93. Dainichi T, et al. Anti-laminin gamma-1 pemphigoid. Proc Natl Acad Sci U S A. 2009; 106:28002805. [PubMed: 19196964]

94. Florea F, et al. Ex vivo pathogenicity of anti-laminin $\gamma 1$ autoantibodies. Am J Pathol. 2014; 184:494-506. [PubMed: 24300951]

95. Chan LS, et al. Bullous systemic lupus erythematosus with autoantibodies recognizing multiple skin basement membrane components, bullous pemphigoid antigen 1, laminin-5, laminin-6, and type VII collagen. Arch Dermatol. 1999; 135:569-573. [PubMed: 10328198]

96. Ben-Yehuda A, et al. The urine of SLE patients contains antibodies that bind to the laminin component of the extracellular matrix. J Autoimmun. 1995; 8:279-291. [PubMed: 7612153]

97. Groth S, et al. Antibodies to the C-terminus of laminin $\gamma 1$ are present in a distinct subgroup of patients with systemic and cutaneous lupus erythematosus. Lupus. 2012; 21:1482-1483. [PubMed: 22968451] 
98. Galvin JE, et al. Cytotoxic mAb from rheumatic carditis recognizes heart valves and laminin. $\mathrm{J}$ Clin Invest. 2000; 106:217-224. [PubMed: 10903337]

99. Ellis NMJ, et al. T cell mimicry and epitope specificity of cross-reactive T cell clones from rheumatic heart disease. J Immunol. 2005; 175:5448-5456. [PubMed: 16210652]

100. McFadden JP, Kimber I. A review on the potential role of basement membrane laminin in the pathogenesis of psoriasis. Scand J Immunol. 2016; 83:3-9. [PubMed: 26346598]

101. Coito AJ, et al. The expression of extracellular matrix proteins during accelerated rejection of cardiac allografts in sensitized rats. Transplantation. 1994; 57:599-605. [PubMed: 8116048]

102. Mueller AR, et al. The extracellular matrix: an early target of preservation/reperfusion injury and acute rejection after small bowel transplantation. Transplantation. 1998; 65:770-776. [PubMed: 9539087]

103. Mueller AR, et al. Changes at the extracellular matrix during acute and chronic rejection in human liver transplantation. Transpl Int. 1998; 11:S377-S382. [PubMed: 9665020]

104. Riederer I, et al. Local antilaminin antibody treatment alters the rejection pattern of murine cardiac allografts: correlation between cellular infiltration and extracellular matrix. Transplantation. 2002; 74:1515-1522. [PubMed: 12490783]

105. Lazarova Z, et al. Passive transfer of anti-laminin 5 antibodies induces subepidermal blisters in neonatal mice. J Clin Invest. 1996; 98:1509-1518. [PubMed: 8833897] 


\section{Trends}

Laminins have an essential role in the formation of basement membranes and in the maintenance of their integrity.

The expression of the laminins is differentially regulated following the induction of tolerance versus immunity and inflammation.

Laminins and especially laminin 411 and 511 isoforms modulate migration and function of the leukocytes.

Targeting immune cell interactions with laminins modulates the induction of immunity or tolerance in autoimmune diseases and transplantation. 


\section{Outstanding Questions}

What are the cellular origins of the laminins in the lymphoid organs? More investigations are necessary to determine the exact origins and locations of the different laminin isoforms in specific tissues.

Which factors (cytokines, proteases, etc.) modulate the expression of laminins in the lymphoid organs? How are these factors regulated?

Which receptors and molecular pathways are involved upon the interaction of immune cell subpopulations with the different laminin isoforms? The important diversity of laminin effects on leukocytes suggests that several laminin receptors are involved.

What are the roles of laminins in the regulation of leukocyte function and in the development of autoimmune diseases and graft rejection? The use of tissueconditional laminin knockouts could define previously unknown roles of different laminin isoform in the modulation of the immune system. 
Trends Box

- Laminins have an essential role in the formation of basement membranes and in the maintenance of their integrity.

- The expression of the laminins is differentially regulated following the induction of tolerance versus immunity and inflammation.

- Laminins and especially laminin 411 and 511 isoforms modulate migration and function of the leukocytes

- $\quad$ Targeting immune cell interactions with laminins modulates the induction of immunity or tolerance in autoimmune diseases and transplantation. 


\section{Outstanding Questions Box}

- What are the cellular origins of the laminins in the lymphoid organs? More investigations are necessary to determine the exact origins and locations of the different laminin isoforms in specific tissues.

- Which factors (cytokines, proteases...) modulate the expression of laminins in the lymphoid organs? How are these factors regulated?

- Which receptors and molecular pathways are involved upon the interaction of immune cell subpopulations with the different laminin isoforms? The important diversity of laminin effects on leukocytes suggests that several laminin receptors are involved.

- What are the roles of laminins in the regulation of leukocyte function and in the development of autoimmune diseases and graft rejection? The use of tissue conditional laminin knockouts could defined previously unknown roles of different laminin isoform in the modulation of the immune system 


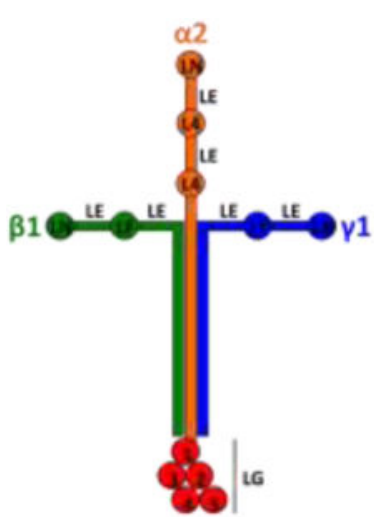

Laminin 211

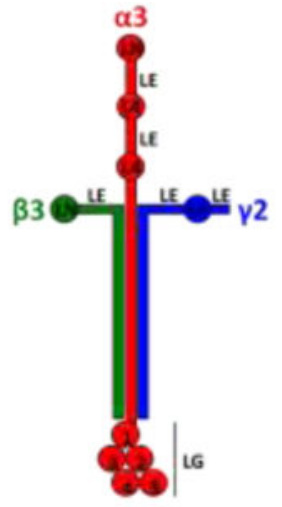

Laminin 332

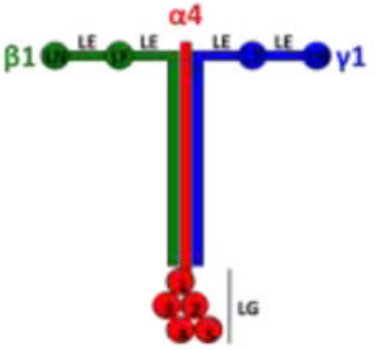

Laminin 411

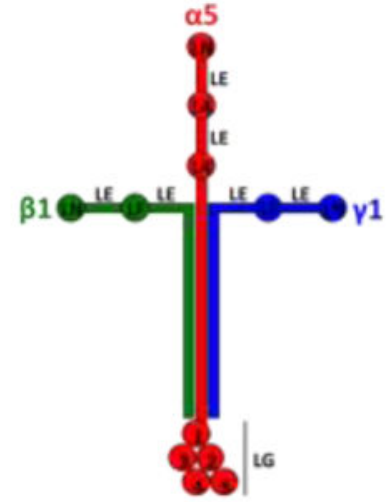

Laminin 511

Figure 1.

Structure of the Four Main Laminins Involved in Immune System Interactions. Greek letters with numbers indicate specific chains. The short arms are composed of a globular laminin N-terminal domain (LN), globular laminin 4 domain (L4), or laminin four domain (LF). Each of these domains is separated by rodlike regions formed by multiple laminin epidermal-growth-factor-like (LE) domains. The C-terminal end is composed of five homologous globular domains (LG) specific to a chains. 


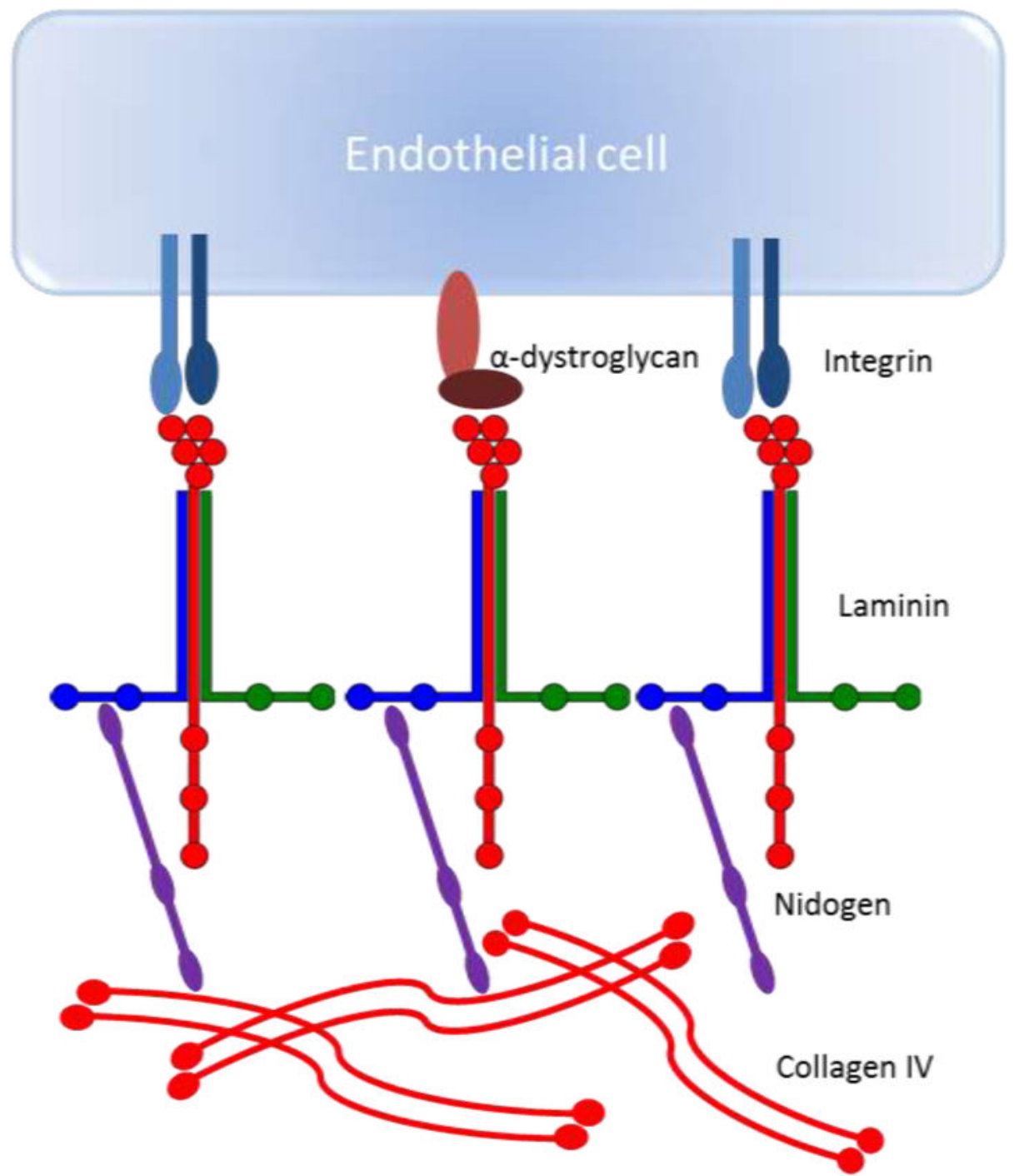

Figure 2.

BM Structure. Laminins allow endothelial cell anchorage to the BM via integrin and adystroglycan receptors. The laminin short arms allow the interaction with nidogen and laminin self-assembly. Nidogen allows the laminin network to be connected to collagen IV. 


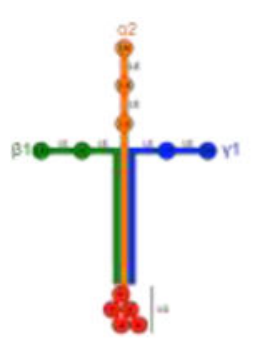

Laminin 211

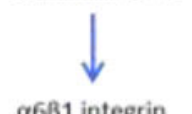

$\alpha 6 \beta 1$ integrin
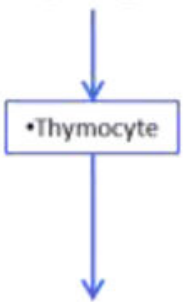

Adhesion Proliferation Cell survival

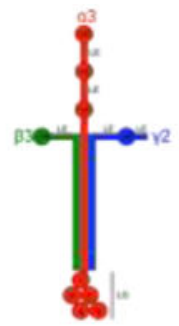

Laminin 332
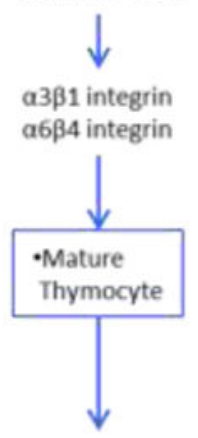

Adhesion

Migration Activation

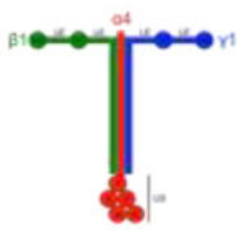

Laminin 411
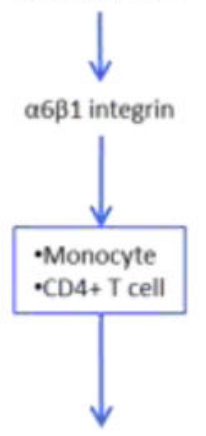

Adhesion Migration

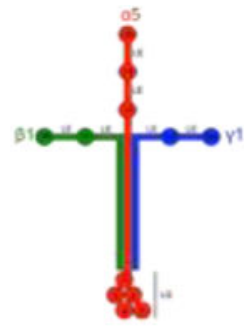

Laminin 511

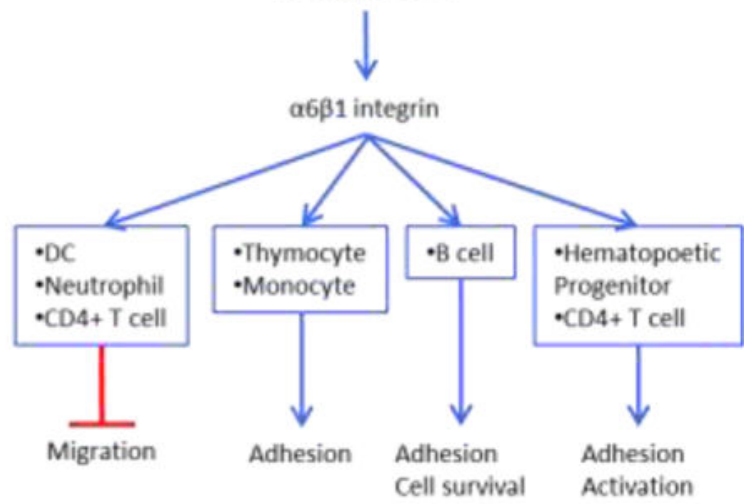

Figure 3.

Laminin and Immune System Interactions. Specific laminin isoforms support and influence the migration and function of distinct immune cells via integrin receptors. DC, dendritic cell; L4, globular laminin 4 domain; LE, laminin epidermal-growth-factor-like; LF, laminin four domain; LG domain, homologous globular domain; LN, globular laminin N-terminal domain. 


\section{Table 1}

Tissue Localization of the Different Laminin Isoforms ${ }^{a}$

\begin{tabular}{|l|c|c|l|}
\hline Isoforms & Abbreviation & Previous abbreviation & Tissue localization \\
\hline$a 1 \beta 1 \gamma 1$ & 111 & 1 & $\begin{array}{l}\text { Parenchymal BM, meningeal epithelium, renal proximal tubule BM, seminiferous } \\
\text { epithelium, adrenal, salivary and mammary gland BM }\end{array}$ \\
\hline$a 1 \beta 2 \gamma 1$ & 121 & 3 & Placenta \\
\hline$a 2 \beta 1 \gamma 1$ & 211 & 2 & $\begin{array}{l}\text { Endoneurium, skeletal and cardiac muscle BM, thymic epithelium, vascular smooth } \\
\text { muscle BM (venule, arteriole), bone marrow }\end{array}$ \\
\hline$a 2 \beta 2 \gamma 1$ & 221 & 4 & Epineurium, neuromuscular junction BM, striated muscle BM \\
\hline$a 3 \mathrm{~A} \beta 3 \gamma 2$ & $3 \mathrm{~A} 32$ & $5 \mathrm{~A}$ & Epithelial BM (keratinocytes), mammary gland BM, placenta, amnion BM \\
\hline $\mathrm{a} 3 \mathrm{~B} \beta 3 \gamma 2$ & $3 \mathrm{~B} 32$ & $5 \mathrm{~B}$ & Epithelial BM (epidermis), thymic medulla \\
\hline $\mathrm{a} 3 \mathrm{~A} \beta 1 \gamma 1$ & 311 & 6 & Epithelial BM (keratinocytes), amnion BM \\
\hline $\mathrm{a} 3 \mathrm{~A} \beta 2 \gamma 1$ & 321 & 7 & Amnion BM \\
\hline $\mathrm{a} 4 \beta 1 \gamma 1$ & 411 & 8 & $\begin{array}{l}\text { Endoneurium, endothelial BM (ubiquitous), vascular smooth muscle BM, bone } \\
\text { marrow, lymph node cortical ridge }\end{array}$ \\
\hline $\mathrm{a} 4 \beta 2 \gamma 1$ & 421 & 9 & Epineurium, neuromuscular junction BM \\
\hline $\mathrm{a} 5 \beta 1 \gamma 1$ & 511 & 10 & $\begin{array}{l}\text { Embryonic BM, endoneurium, epithelial BM, endothelial BM (ubiquitous), vascular } \\
\text { smooth muscle BM, bone marrow, lymph node cortical ridge, spleen MZ }\end{array}$ \\
\hline $\mathrm{a} 5 \beta 2 \gamma 1$ & 521 & 11 & Epineurium, neuromuscular junction BM, renal glomerular endothelium \\
\hline $\mathrm{a} 2 \beta 1 \gamma 3$ & 213 & 12 & Placenta \\
\hline $\mathrm{a} 4 \beta 2 \gamma 3$ & 423 & 14 & Retinal BM, hippocampal synapse \\
\hline $\mathrm{a} 5 \beta 2 \gamma 2$ & 522 & - & Bone marrow \\
\hline $\mathrm{a} 5 \beta 2 \gamma 3$ & 523 & 15 & Retinal BM \\
\hline
\end{tabular}

${ }^{a}$ Sixteen laminin isoforms have been described and their distribution is tissue specific. Every BM contains at least one laminin isoform that dictates its architecture and properties. 
Table 2

Modulation of Immune Cell Functions by Laminins ${ }^{a}$

\begin{tabular}{|c|c|c|c|c|}
\hline Immune cells & Laminins & Laminin receptors & Laminin effects & Refs \\
\hline Hematopoietic progenitors & $211,411 / 421,511 / 521$ & Integrin a $6 \beta 1$ & Adhesion, migration, proliferation & {$[34-36,52,53]$} \\
\hline Thymocytes & $211,511,332$ & $\begin{array}{l}\text { Integrins a } 6 \beta 1, a 3 \beta 1 \text {, } \\
\text { a } 6 \beta 4\end{array}$ & $\begin{array}{l}\text { Adhesion, migration, maturation, } \\
\text { reduction of apoptosis, activation of } \\
\text { MMP-14 }\end{array}$ & {$[37,54-59]$} \\
\hline Neutrophils & $111,332,411,511$ & Integrins a $6 \beta 1$ & $\begin{array}{l}\text { Adhesion, migration, increased TNF- } \\
\text { a, MIP- } 1 \beta \text {, lysozyme, superoxide } \\
\text { production, and bactericidal activity }\end{array}$ & {$[7,41,46,60-69]$} \\
\hline Basophils & 111 & Integrin a $6 \beta 1$ & Adhesion & {$[70,71]$} \\
\hline Eosinophils & 111 & Integrin a $6 \beta 1$ & $\begin{array}{l}\text { Adhesion, increased viability, } \\
\text { reduced activation, and degranulation }\end{array}$ & [72-74] \\
\hline Mast cells & 332,511 & Integrins $a 6 \beta 1, a 3 \beta 1, a 7$ & $\begin{array}{l}\text { Adhesion, migration, increased IL- } 8 \\
\text { production }\end{array}$ & [75-78] \\
\hline Macrophages & 111,511 & Integrin a $6 \beta 1$ & $\begin{array}{l}\text { Adhesion, increased urokinase-type } \\
\text { plasminogen activator, MMP- } 9 \text {, TNF- } \\
\text { a production, and phagocytic } \\
\text { function }\end{array}$ & [79-86] \\
\hline Monocytes & $111,411,511$ & Integrins $a 6 \beta 1, \beta 2$ & $\begin{array}{l}\text { Adhesion, migration, increased TNF- } \\
\text { a, IL- } 1 \beta, \text { IL- } 6 \text {, MMP- } 9 \text { production, } \\
\text { and phagocytic function }\end{array}$ & {$[42,64,83,87]$} \\
\hline DCs & $111,411,511$ & Integrin $\beta 1$ & $\begin{array}{l}\text { Adhesion, migration, increased } \\
\text { phagocytic function }\end{array}$ & {$[6,88-90]$} \\
\hline B lymphocytes & 511 & Integrin a $6 \beta 1$ & Adhesion, migration, survival. & {$[38]$} \\
\hline T lymphocytes & $111,411,511$ & Integrin a $6 \beta 1, \mathrm{MCAM}$ & $\begin{array}{l}\text { Adhesion, migration, activation, } \\
\text { proliferation }\end{array}$ & {$[6,44,45,47,82]$} \\
\hline
\end{tabular}

${ }^{a}$ Almost all leukocyte subpopulations express specific laminin receptors. Laminins act as ligands that bind these cell membrane receptors, initiating signaling that modulates leukocyte functions and migration. 